\title{
SOME OBSERVATIONS ON THE SURGICAL TREATMENT OF. CONCOMITANT STRABISMUS
}

\author{
BY \\ Howard Price Folger, M.D. C.M. \\ KINGSTON, ONTARIO
}

IN the surgical treatment of concomitant strabismus any procedures which will aid in deciding with which muscle or muscles we should deal and which operation or operations should be chosen, are of advantage. Anything that will assist in making results more exact, or that will simplify or smooth the way, for operator or patient, is to be desired. With this in yiew this paper presents certain points, most of which are not new but some of which are often forgotten. Many of these points have been stressed from time to time by White, Jameson and others to whom I am indebted.

The first requisite in dealing surgically with a case of strabismus is a thorough pre-operative examination. This should be comprehensive enough to include:

1. An estimation of the visual acuity, and refraction under a cycloplegic.

2. Estimation of the near point of convergence.

3. Estimation of the deviation for distance and near in the six cardinal fields.

4. Estimation by the screen comitance test of the ocular motility and any associated vertical deviation.

Estimation of Convergence Near Point.-The near point of convergence may be estimated by utilizing a small spot on a card (or the head of a pin) as a point of fixation. This is brought toward the eyes and the point at which the non-fixing eye diverges (relative convergence near point) is noted.

The measurement is made along the intracentral base line from the anterior focal point $(13.5 \mathrm{~mm}$. in front of the cornea). As the centre of rotation is $11.5 \mathrm{~mm}$. behind the cornea, $25 \mathrm{~mm}$. should be added to the amount as measured by the millimetre rule.

Estimation of Deviation for Distance and Near in the Six Cardinal Fields.-By Screen Test.-To estimate the deviation for distance the patient is made to fix at 20 feet. A card is then passed across his face; covering first one eye, then the other. In convergent squint, prisms, base out, are placed before either eye until no movement outward can be detected when the card is moved from one eye to the other. Divergent squint is measured 
by prisms placed base in and hypertropia by prisms base down before the higher eye (or base up before the lower).

The test is then repeated with fixation at $33 \mathrm{~cm}$.

Next, fixation is made in each of the six cardinal fields and the deviation measured by prisms in the same way.

\section{Screen Comitànce Test}

The screen comitance test will give further information as to paralysis, contractures and secondary deviations. In making this test a card is held so that the patient sees the object of fixation first with one eye, then with the other, while the examiner watches both. It is carried out in the six cardinal fields.

\section{Hirschberg's Test}

In cases of squint in young children who will not fix the test object Hirschberg's test is useful. A small electric light is held about a foot in front of the patient's face and the position of the light reflex on the cornea noted. If this is at the temporal edge of the pupil squint is about 15 degrees; if midway between margin of pupil and limbus, about 30 degrees; if at limbus about 45 degrees.

In adults, where vision of one eye is very poor or a central scotoma makes fixation difficult, perimeter measurement of the deviation, or Hirschberg's test may be used. Although not as accurate as prism measurement, still from a practical standpoint, perimeter measurement of the deviation plus a careful study of the other factors involved, will give an excellent background on which to base operative procedures.

\section{Choice of Operation}

With the above information at hand we are in a position to form some opinion as to the type of squint and which muscles should be surgically altered. Where possible, the muscles at fault are those with which we should deal.

In cases of convergence excess some form of operation to reduce the effectiveness of the interni is indicated. My preference here is for the Jameson -recession operation; rather than tenotomy. Thus, if the squint is more marked for near than for distance, and if it is reduced by the instillation of a mydriatic and with a plus correction we know that it belongs to the convergence excess type and recession of one or both interni is indicated. Along with this we should consider the distance of the convergence near point. The closer the convergence near point, the more the power of 
the interni should be reduced and the farther off it is the less should be the interference.

In cases where the near point is very remote (say $80-90 \mathrm{~mm}$.) no operation which would weaken the action of the interni should be undertaken. In this case operation on the externi only should be done.

In convergent squint, cases of divergence insufficiency will show themselves by the squint being greater for distance than for near, or about the same. In these cases operation should be designed to increase the power of the weakly acting externi.

In many instances both weakening of the interni and strengthening of the externi may be indicated, but the interni should never be weakened more than the amount of the convergence near point would seem to allow.

White* has stated that vertical deviations if marked, should be corrected first, except where lateral strabismus is much greater than the vertical and is not lessened by prismatic correction of the vertical deviation in making tests.

The actual operation chosen depends greatly on the preference of the individual operator. Too much stress has been laid at times on minor differences in suturing and incisions and the broad principles neglected. To increase the acting strength of a muscle I prefer a resection operation ordinarily. If the muscle is very attenuated and feeble I choose a capsulo-muscular advancement as a rule, often with resection. For weakening the action of a muscle the Jameson recession operation serves admirably, being far superior to and more accurate than the ordinary tenotomy.

\section{Amount of Correction}

It is usually stated that in squint a deviation of $1 \mathrm{~mm}$. equals 5 degrees of arc. This is arrived at by taking the diameter of the eyeball as $23 \mathrm{~mm}$. which gives us about $72 \mathrm{~mm}$. in the circumference. As there are 360 degrees in the circumference this works out to $1 \mathrm{~mm}$. equals 5 degrees of arc. Since 5 degrees of arc is also equal to 10 prism dioptres, it follows that $\mathrm{I} \mathrm{mm}$. deviation also equals 10 prism dioptres. Thus, if we know the amount of the deviation in degrees or prism dioptres, we can estimate the amount of shift of the eyeball, in millimetres necessary to correct it.

If the eye is larger than normal $1 \mathrm{~mm}$. deviation would represent less than 5 degrees. In smaller eyes more than 5 degrees. It must be remembered that linear shortening or lengthening of one muscle by $1 \mathrm{~mm}$. for example does not necessarily result in $1 \mathrm{~mm}$. shift of the globe.

* White, J. W.-When and how should one operate for Convergent Strabismus. Arch. Ophthal., Vol. XII, p. 699, November, 1934 . 
Jameson* estimates for the recession operation that $1 \mathrm{~mm}$. recéssion of the internus will correct 5 degrees of deviation. With. a $5 \mathrm{~mm}$. recession of the internus he expects $20^{\circ}-25^{\circ}$ correction and $40^{\circ}-50^{\circ}$ if bilateral.

Recession of the externus gives only about $1 / 3$ as much correction (15-20 for bilateral recession).

Resection or advancement of one externus averages about 15 degrees.

The amount of correction figured beforehand may have to be modified to some extent depending upon local conditions, found when the muscles are exposed.

\section{Age for Operation}

There is nothing to be gained and often much to be lost by waiting too long before operating. The delays recommended by many were based on the idea that one must wait until the patient is old enough to have operation performed under local anaesthesia. It seems reasonable to operate when more conservative measures have proved inadequate. Early operation will often avoid complications due to secondary changes in the muscles. Children with squint are apt to develop an inferiority complex which makes it advisable to correct the condition before the child has to take up competitive work in school.

\section{Anaesthesia}

While it is true that under local anaesthesia one may be able to judge the effect of the procedure and grade the operation accordingly, it is true only under partial anaesthesia which is nerve racking and painful for the patient. In order to secure adequate local anaesthesia a retro-bulbar block is necessary to avoid pain due to traction on the muscles: . This, of course, interferes with the muscular motility and we are no better off than with a general anaesthetic. Further, the healing is not so rapid and the reaction is greater if a local anaesthetic is used. My preference is for general anaesthesia; intra-tracheal, which leaves the area of operation entirely free. With this method the patient can be kept down to the level desired without fear of coming up at an awkward time and is entirely relieved of the pain and mental anguish which so often accompanies inadequate anaesthesia. It is requisite for general anaesthesia, however, that a proper pre-operative examination of the squint be done, and a careful evaluation of the kind and degree of the surgical interference be made.

* Jamieson, P. Chalmers. - The Surgical Entity of Muscle Recession. Arch. Ophthal., Vol. VI, pp. 329-361, September, 1931. 


\section{Sutures}

The use of chromic catgut sutures and atraumatic needles simplifies the operation and adds to the comfort and happiness of the patient. Triple -0 or Four -0 ten day chromic sutures on atraumatic needles will be found entirely satisfactory for all muscle work. These sutures do not have to be removed later and, unlike silk, they become less irritating the longer they remain. The catgut tends to swell and increase in size and for this reason holds well without cutting through.

\section{Recession Operation}

At this point I would like to say a few words about the recession operation as advocated and practised by $\mathrm{P}$. Chalmers Jameson.

This operation is mechanically better, safer, and more precise than the older tenotomy. It gives all the advantages of tenotomy, plus a precise correction with fixation to the sclera which prevents later divergence or caruncular retraction.

The operation has been so often and so ably described at different times by Dr. Jameson that I need not enter here into details of the technique. I have used it in my practice for thirteen years, both alone and in conjunction with advancement and resection. If care is used in placing the sutures, one need have no fear of damage to the eyeball. As Dr. Jameson has pointed out, the needles should be of a type that will split the scleral fibres easily. Small bundles of fibres should be picked up by the point of the needle as it is worked through and the needle and suture should be visible always through the over-lying sclera. If this is observed penetration of the globe need not be feared.

In order to prevent faulty convergence following recession, the muscle should not be receded beyond the equator of the eyeball. This allows us about $5 \mathrm{~mm}$. maximum for recession in the average eye on the internus and about $2.5 \mathrm{~mm}$. for the externus. This rule may be violated occasionally in the case of the externus. If the cornea measures more than $11 \mathrm{~mm}$. the eye is probably larger than normal and a slightly greater amount of recession may be admissible. If the cornea is less than $11 \mathrm{~mm}$. slightly less would be the margin of safety. In some instances the muscle insertions may be nearer than normal to the cornea and in these cases we would have a correspondingly greater distance to recede the muscles before the equator is reached. If insertions are further back than normal a lesser amount of recession would place the muscle ends at the equator.

Broadly speaking, where an internal squint is of a convergence excess type recession of the interni is indicated either single or bilateral. 
In an internal strabismus where convergence is weak and the near point is farther away than normal recession would be inadvisable. In these cases advancement or resection of the externi is indicated.

In many instances these may be combined. When both recession and resection or advancement are used it seems better to do the recession first. This puts less stress on the sutures for the resection with less likelihood of siipping or cutting out.

The cosmetic results with properly performed recession are excellent and there is no faltering of convergence, caruncular retraction, or late deviation outwards.

\section{Post-Operative Treatment}

With recession alone the reaction is usually slight. With advancement or resection it is greater. Ice compresses during the early stage give comfort and reduce swelling. The eyes should be irrigated with boracic solution and the dressing changed once or twice daily. Metaphen ointment or some other antiseptic is instilled when dressings are changed, and atropine 1 per cent. used daily. Both eyes are covered for the first few days. In recession alone one eye is uncovered about the third day and both about the fifth or sixth. With advancement both eyes are kept covered for about 5 days. (Orthoptic treatment during the early healing period does more harm than good.)

To summarise, the value of a careful pre-operative examination in strabismus has been stressed. With this as-a background plus an evaluation of the local conditions found at the time of operation, results under general anaesthesia are good. Operation should be performed when other treatment has failed and not delayed too long. Recession of the over-acting muscles or advancement or resection of the under-acting muscles, or a combination of these, will correct most cases. The recession operation is preferable to tenotomy.

\section{ANNOTATION}

\section{Disability Lawsuit following Successful Bilateral Cataract Extraction}

This, is the title of a short paper in the Amer. Jl. of Ophthal. February, 1942, read before the Colorado Ophthalmological Society at Denver in November, 1940, by R. W. Danielson and J. C. Long. 